\title{
Short communication \\ Electrospun fibre colorimetric probe based on gold nanoparticles for on-site detection of $17 \beta$-estradiol associated with dairy farming effluents
}

\author{
Bellah O Pule ${ }^{1}$, Slyvestre Degni ${ }^{1}$ and Nelson Torto ${ }^{1 *}$ \\ 'Department of Chemistry, Rhodes University, PO Box 94, Grahamstown 6140, South Africa
}

\begin{abstract}
An on-site colorimetric probe, based on gold nanoparticles incorporated into electrospun polystyrene nanofibres, for the detection of oestrogenic compounds, as represented by $17 \beta$-estradiol, in dairy effluents is presented. The probe exhibited a significant absorption peak at $542 \mathrm{~nm}$, ascribed to surface plasmon resonance of Au nanoparticles (NPs). With increasing $17 \beta$-estradiol concentration the surface plasmon resonance (SPR) band shifted to a longer wavelength accompanied by a visual colour change from shades of pink to blue. The visible cut-off concentration was $100 \mathrm{ng} / \mathrm{m} \ell$. Upon exposure to cholesterol and a series of compounds known to induce oestrogenic activity, p,p'-DDE, deltamethrin, 4-tert-octylphenol and nonylphenol, only $17 \beta$-estradiol could induce a pink colour observable by the naked eye, which is indicative that the proposed gold nanoparticles-incorporated electrospun polystyrene nanofibres could be employed as highly selective colorimetric strips to detect $17 \beta$-estradiol, with minor interference from other endocrine-disrupting compounds usually present in dairy effluents. The facile nature of the colorimetric probe and potential application in monitoring water quality was demonstrated.
\end{abstract}

Keywords: $17 \beta$-estradiol, wastewater, colorimetric probe, gold nanoparticles, aggregation, dispersion

\section{INTRODUCTION}

Dairy-farming wastewater contains significant concentrations of natural steroidal oestrogens such as $17 \alpha$-estradiol, $17 \beta$-estradiol, and estrone, which can potentially contaminate surface and ground water (Hanselman et al., 2006; Zheng et al., 2008). Oestrogenic contamination of surface waters is of concern for fish as it is associated with feminisation of male fish, reproductive abnormalities and skewed sex ratios (Oishi, 2010; Gadd et al., 2010). Within the oestrogenic contaminants, $17 \beta$-estradiol $\left(\mathrm{E}_{2}\right)$ has emerged as a target for recognition and analysis due to its dual role (Chiu et al., 2008). $17 \beta$-estradiol plays an important function during the various stages of mammalian development, including growth and reproduction. However, $\mathrm{E}_{2}$ is also the most potent naturallyoccurring oestrogen as it has the highest oestrogenic activity at $\mathrm{ng} / \ell$ concentrations (Hanselman et al., 2003). Although several detectors, in particular, flame ionisation or mass spectrometric detection coupled with GC, have had significant achievements in the determination and quantification of oestrogenic steroid hormones in aqueous samples (Wang, 2011), they are expensive, sophisticated and require extensive sample handling. Owing to the current detection drawbacks, simple, rapid, reliable and cheap protocols are highly desirable. Technically, the non-invasive analytical devices based on colorimetric principles not only take advantage of colour for rapid detection but also allow for

\footnotetext{
To whom all correspondence should be addressed.

Iiir +27 76061 4005; fax: +27 46622 5109;

e-mail: n.torto@ru.ac.za; nelsontorto@yahoo.co.uk

Received 10 May 2012; accepted in revised form 20 November 2014.
}

simplicity as they are cost effective (Chigome and Torto, 2011). Specifically, metallic nanoparticle-based colorimetric assays allow for on-site detection (minimising sample handling) rendering both quantitative and qualitative analysis in biological, biotechnological or environmental matrices amenable (Ma et al., 2011).

Of particular interest are the gold nanoparticles (Au NPs)based colorimetric approaches as they take advantage of the surface plasmon resonance (SPR) dependent colour changes (Romeo et al., 2012; Zhao et al., 2008). Gold nanoparticles exhibit distinct and well-defined colours that are easily perceptible by the naked eye (Jones et al., 2011; Jans and Huo, 2012; Saquing et al., 2009). Generally, the colorimetric mechanisms for Au NPs are based on their aggregation or dispersion with guest molecules, i.e., analytes (Ding et al., 2012). In principle, there should be a balance between the inter-particle attractive and repulsive forces. The relationship between these forces determines whether Au NPs are stabilised or aggregated and the resulting solution colour is central to the design of $\mathrm{Au}$ NP-based colorimetric systems (Zhao et al., 2008). However, in solution, Au NPs have a tendency to aggregate, particularly in the presence of salts and some biological molecules such as proteins (Gao et al., 2012). To address the instability of Au NPs in solution, polymers may be integrated into the system (Mayer, 1998).

Several features play an important role in the integration of polymers into nanocomposite systems. Two important aspects to consider, as highlighted in a review by Grubbs, are how the polymer ligands affect accessibility of the inorganic nanoparticle surface, and compatibility of the entire polymer-coated nanoparticle with a broad variety of other materials (Grubbs, 2007). The nanocomposites may be used in solution (Zhang et 
al., 2010) or in a solid platform (Bai et al., 2008). Various useful methods have been developed to fabricate hybrid organicinorganic systems, for example, Langmuir-Blodgett assembly, layer-by-layer assembly, chemical deposition and spin-coating (Lu et al., 2005). However, these approaches are often challenged when it comes to producing stable composites. In this study, a facile electrospinning technique is employed for the fabrication of polystyrene-gold polymer composite fibres. The electrospun composite fibres were employed as test strips for the colorimetric detection of $17 \beta$-estradiol. Upon interaction with the analyte, the white fibre mat changed colour to either a shade of blue or pink, at higher and lower concentrations of $17 \beta$-estradiol, respectively.

\section{EXPERIMENTAL}

\section{Materials}

Polystyrene ( $\left.\mathrm{M}_{\mathrm{w}}=192000\right)$, gold (III) chloride trihydrate $\left(\mathrm{HAuCl}_{4} \cdot 3 \mathrm{H}_{2} \mathrm{O}\right)$ and sodium borohydride $\left(\mathrm{NaBH}_{4}\right)$, all of purity $>99 \%$, were purchased from Sigma-Aldrich, (St. Louis, MO, USA). All reagents were of analytical or HPLC grade. Tetrahydrofuran, THF (98\%) and N, N-dimethylformamide, DMF (99\%) were purchased from Merck Chemicals (Wadeville, South Africa), and methanol (MeOH) from Merck KGaA (Darmstadt, Germany), while formic acid and glacial acetic acid (HAc) were from Sigma-Aldrich (St. Louis, MO, USA). $17 \beta$-estradiol, p,p'-DDE (1,1-dichloro-2,2-bis(p-chlorophenyl) ethylene), deltamethrin (D), 4-tert-octylphenol, nonylphenol and cholesterol were also purchased from Sigma-Aldrich. Primary steroid, pesticides, alkylphenols and cholesterol stock solutions were each prepared at nominal concentrations of $1000 \mu \mathrm{g} / \mathrm{m} \ell$ in $\mathrm{MeOH}$.

\section{Synthesis of Au-PS composite}

In a typical procedure, predetermined quantities of PS and $\mathrm{Au}$ salt were dissolved in DMF/THF (8:2 v/v) in a sealed vial. The PS mixture was left to stir for 3 to $4 \mathrm{~h}$ to ensure complete dissolution, after which $\mathrm{NaBH}_{4}$ was added to effect the reduction of the metal salt precursor at ambient conditions. The solution was left to stir overnight to eliminate air bubbles and then was electrospun. The electrospinning set-up included a syringe pump operated at a flow rate of $0.300 \mathrm{~m} / \mathrm{h}$ and a high-voltage power supply with a positive polarity. The optimum voltage applied was $22.2 \mathrm{kV}$.

\section{Characterisation of the polymer composites}

Polystyrene-gold composite solution was characterised employing the transmission electron microscope (TEM) prior to electrospinning. The morphology of the fibres was evaluated with a Jeol JSM - $700 \mathrm{~F}$, field emission scanning electron microscope (FE-SEM) operating at $30 \mathrm{kV}$ after gold coating of the sample. UV-visible absorption spectra were recorded using a Perkin Elmer Lambda $25 \mathrm{UV} / \mathrm{Vis}$ spectrophotometer.

\section{Matrix spike experiments}

The dairy effluents were collected from a local cattle farm. Grab samples were collected from the milking shed drains and preserved by adding concentrated sulphuric acid ( $\mathrm{pH} 3)$. The dairy water samples were stored at $<4^{\circ} \mathrm{C}$ overnight, then thawed gradually to room temperature and used within $24 \mathrm{~h}$.

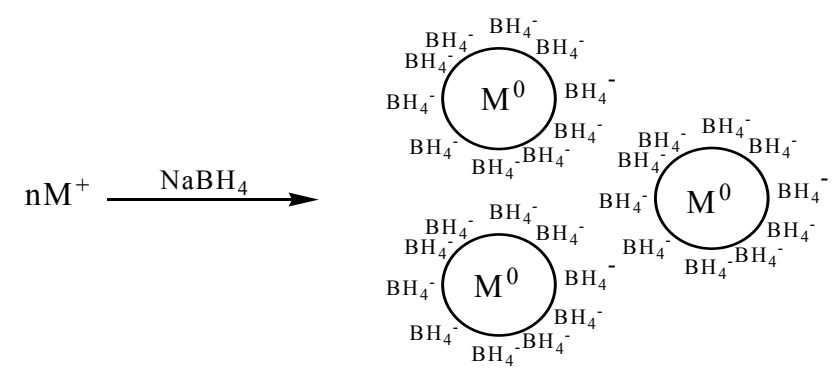

Figure 1

Schematic illustration for synthesis and stabilisation of metal nanoparticles by tetrahydroborate ions $\left(\mathrm{BH}_{4}^{-}\right)$ [adapted from Olenin and Lisichkin, 2011]

The sample matrix was spiked with $17 \beta$-estradiol and other environmental chemicals with oestrogenic activity, including p,p'-DDE, deltamethrin, 4-tert-octylphenol and nonylphenol, as well as with cholesterol, so as to evaluate the colorimetric response of the Au-PS NP probe strips.

\section{RESULTS AND DISCUSSION}

\section{Synthesis of Au-PS composite}

Polystyrene-stabilised gold nanoparticles were synthesised by in-situ reduction of $\mathrm{Au}^{3+}$ with sodium borohydride. Sodium borohydride has been the choice of reducing agents for $\mathrm{Au}$ and Ag nanoparticles because of its greater reducing power than other reducing agents, such as hydrazine and ascorbic acid, which give larger nanoparticles (Seo et al., 2009). It has been shown that in a system where species do not have a high affinity towards $\mathrm{Au}, \mathrm{BH}_{4}^{-}$ions bind to the surface of gold nanoparticles and form a negatively charged layer that contributes towards stabilising the nanoparticles (Fig. 1). However, due to secondary interactions with the environment, $\mathrm{BH}_{4}$ - ions are generally not efficient stabilisers (Olenin and Lisichkin, 2011; Uehara, 2010). For instance, $\mathrm{BH}_{4}$ - ions adsorbed on the surface of nanoparticles usually react with water molecules resulting in aggregation of nanoparticles. For this study polystyrene was used to stabilise the nanoparticles against aggregation.

Polymers (e.g. polystyrene), including small molecules and polyelectrolytes, have been commonly used to stabilise nanoparticles through steric, electrostatic and electrosteric (a combination of electrostatic and steric) interactions, respectively (Zhang et al., 2011).

\section{Characterisation of the polymer composites}

The prepared PS-Au NPs showed an absorption peak at 542 $\mathrm{nm}$, which was ascribed to the surface plasmon resonance of $\mathrm{Au}$ NPs. The interaction of $\mathrm{E}_{2}$ with PS-Au NP-induced aggregation of PS-Au NPs and resulted in the SPR absorption band shifting to a longer wavelength (Fig. 2A). The TEM images exhibited spherical and triangular plates PS-Au NPs (Fig. 2B) while the $17 \beta$-estradiol-stimulated aggregation of PS-Au NPs was further verified in Fig. 2C.

Polystyrene-gold nanoparticle composite solutions were electrospun to give white fibre mats. In order to attain intense changes in colour of PS-Au NPs in response to $E_{2}$, the effect of concentration of Au NPs in polymer solution on the morphology of the electrospun fibres was investigated. The nanoparticle 

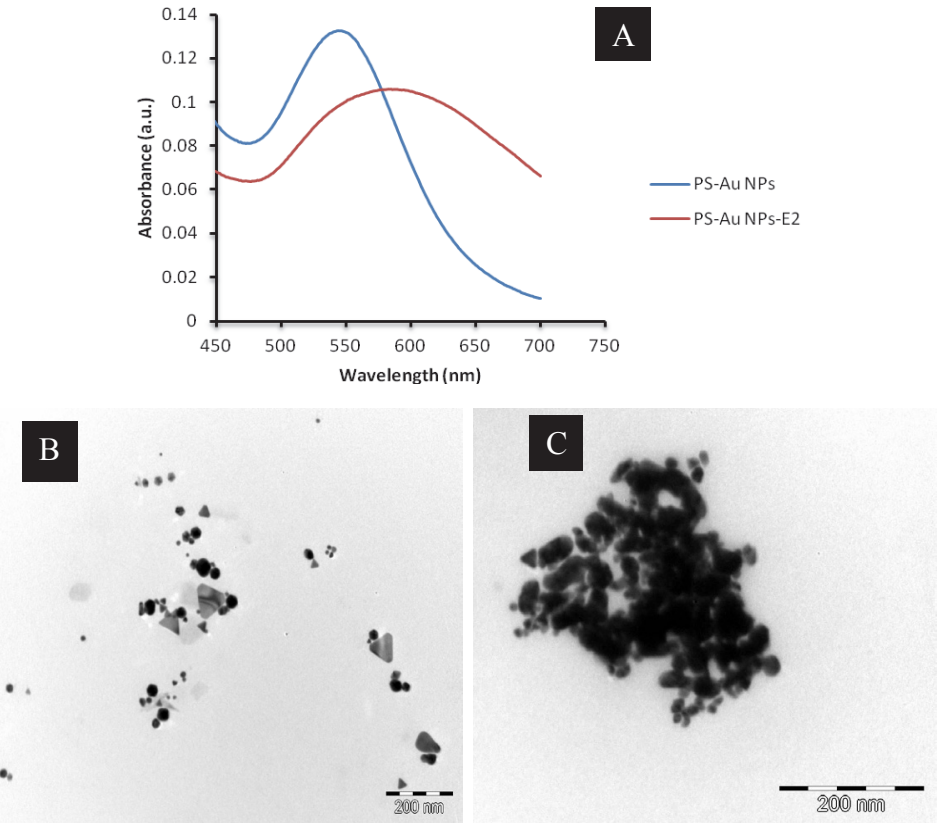

Figure 2

(A) Typical absorption spectra of the PS-Au NPs in the absence and in the presence of $17 \beta$-estradiol (PS-Au NP-E $)$; typical TEM images of PS-Au $N P s(B)$ in the absence and

(C) in the presence of $17 \beta$-estradiol (PS-Au NP-E2)
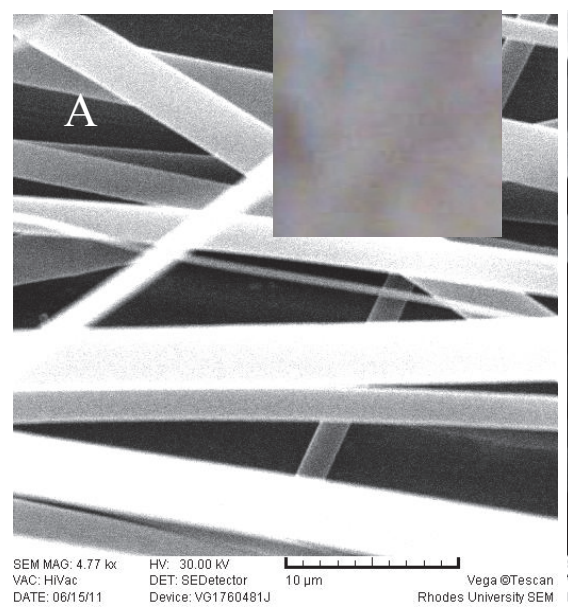
SEM MAG: 4.77 W
VAC: Hiva
DATE: 0615 5:11

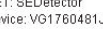

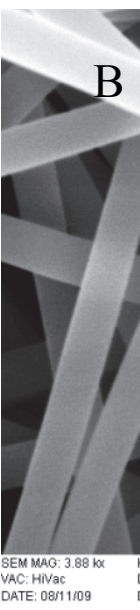

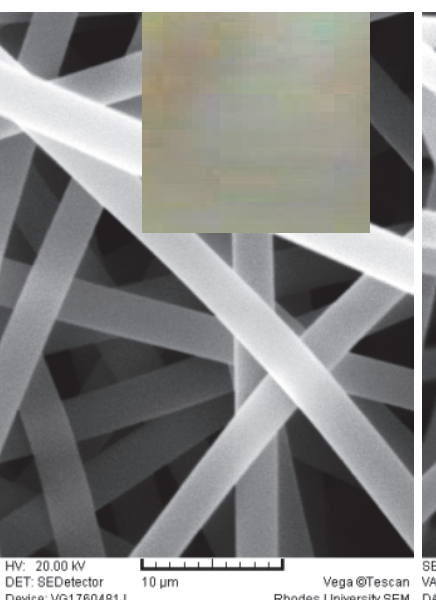

DET: SEDetector
Device: Vo1760481,

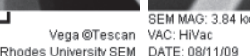

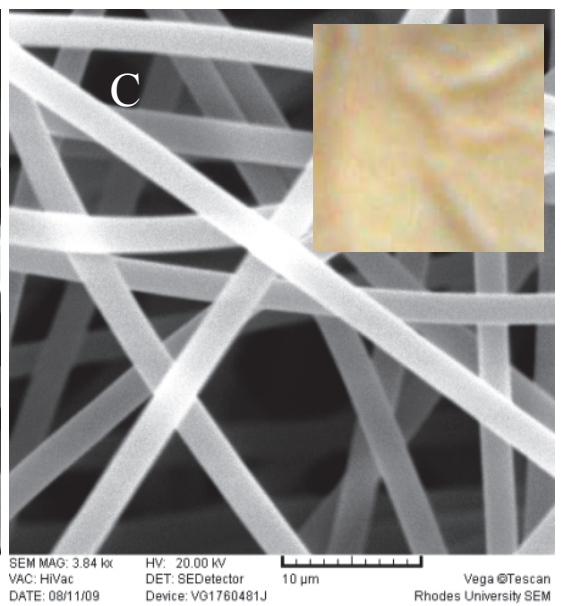

Figure 3

Scanning electron microscope images of $15 w t \%$ PS-Au NP electrospun fibres at ratio (A) 1:40; (B) 1:20; (C) 1:10. The inserts show corresponding photographs of PS-Au NP electrospun fibres upon interaction with $100 \mathrm{ng} / \mathrm{ml} 17 \beta$-estradiol

to polystyrene weight ratios were 1:40, 1:20 and 1:10. The morphology of the PS-Au NP electrospun fibres was observed with SEM. The scanning electron microscope images showed a decrease in fibre diameter with increasing concentration of $\mathrm{Au}$ NPs (Fig. 3). The observed decrease in diameter of the PS-Au NP composite nanofibres with increasing Au NP content may be due to the increased conductivity of the nanocomposite solutions (Kim and Ahn, 2008). The ratio 1:40 gave the best colour changes (Fig. 3B insert), and was therefore chosen to give the optimum Au NP concentration.

At a higher magnification, a high-resolution SEM image confirms the non-beaded morphology of electrospun PS-Au NP composite fibres (Fig. 4). The insert in Fig. 4 exhibits encapsulated and dispersed Au NPs within the PS electrospun fibre. The assumption is that the Au NPs are dispersed as small clusters rather than as individual nanoparticles. The instability of the gold nanoparticle clusters drove the colorimetric mechanism for the naked eye detection of $17 \beta$-estradiol.

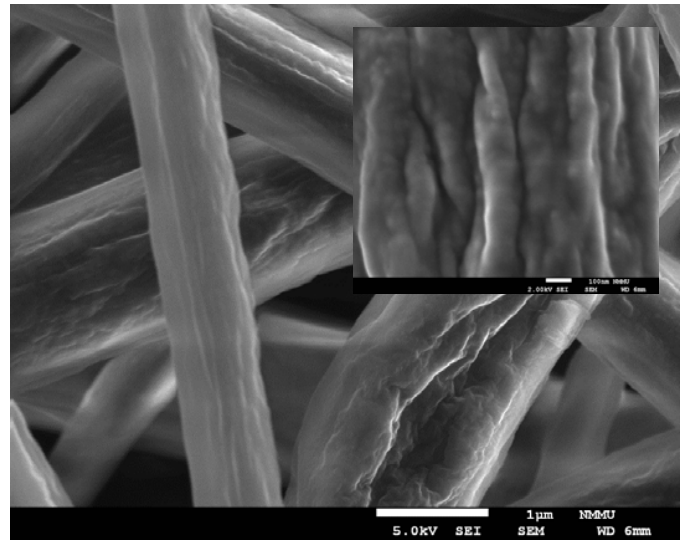

Figure 4

HRSEM image of PS-Au fibres electrospun from 15 wt\% PS with molar ratio of $40: 1$ 


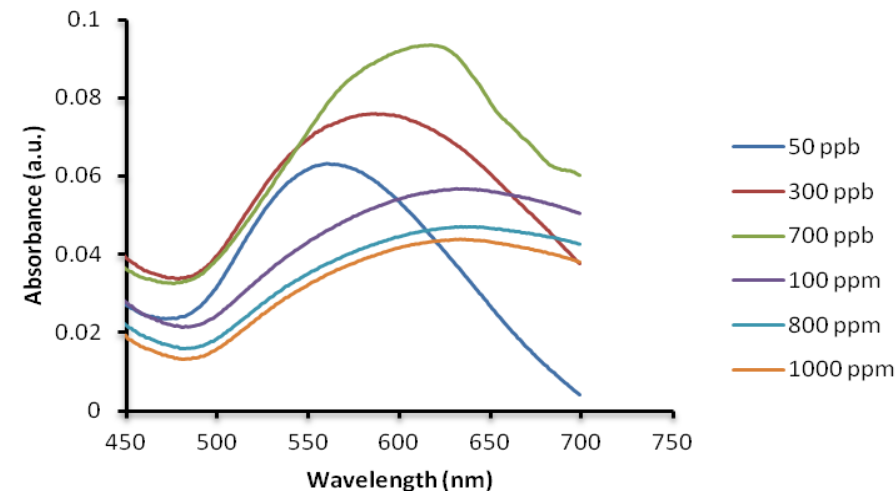

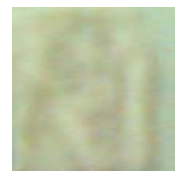

Free test strip

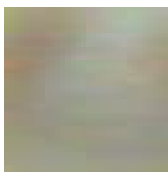

$50 \mathrm{ng} / \mathrm{m \ell}$

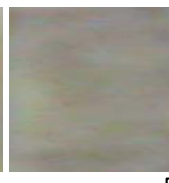

$\longrightarrow$
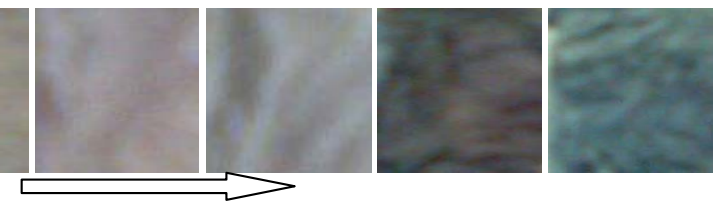

$1000 \mu \mathrm{g} / \mathrm{m \ell}$
Figure 5

UV-vis absorbance spectra and photographs showing PS-Au NP probe strips upon exposure to increasing $17 \beta$-estradiol concentrations $(50 \mathrm{ng} / \mathrm{m \ell}$ $300 \mathrm{ng} / \mathrm{m \ell}, 700 \mathrm{ng} / \mathrm{m \ell}$, $100 \mu \mathrm{g} / \mathrm{ml}, 800 \mu \mathrm{g} / \mathrm{ml}$ and $1000 \mu \mathrm{g} / \mathrm{ml}$ ) at 60 s exposure time for each concentration
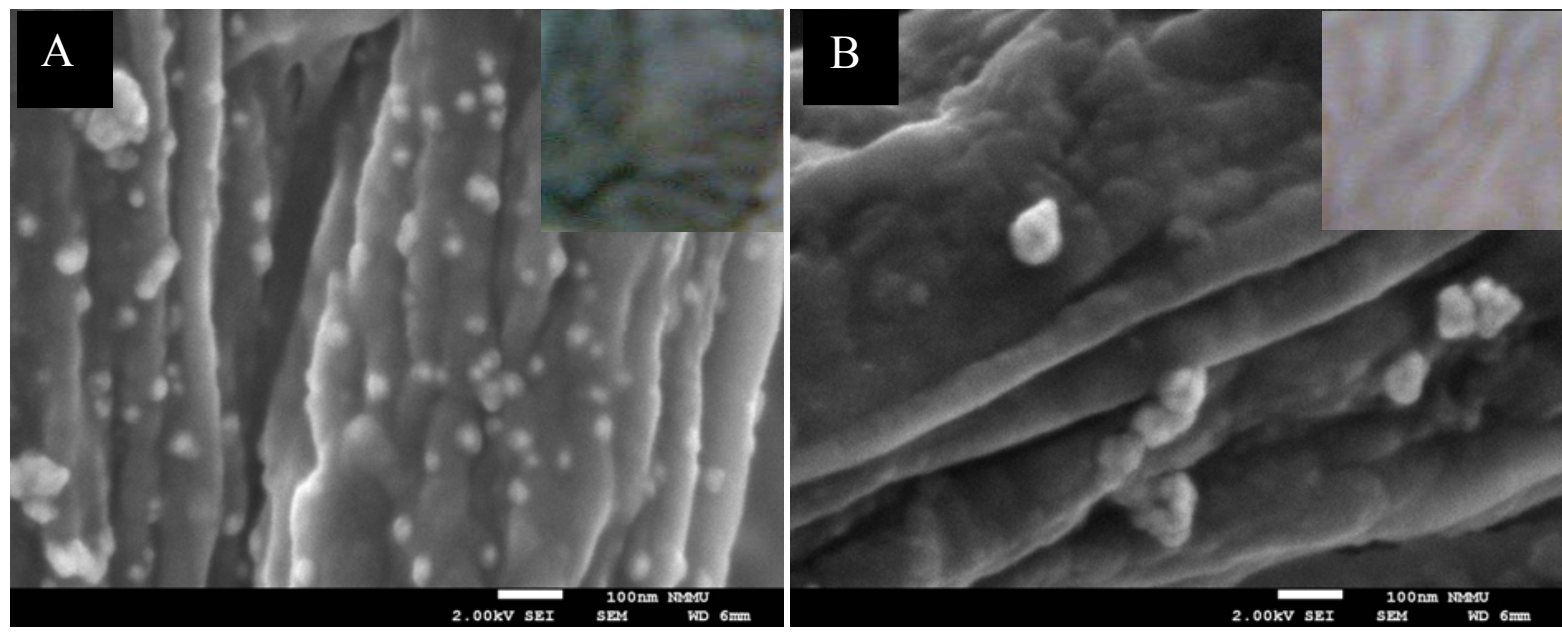

Figure 6

HRSEM images of (A) Au NP agglomerates after interacting with $E_{2}$ at $800 \mu \mathrm{g} / \mathrm{ml}$ (inset is a typical blue probe indicative of higher concentrations of $E_{2}$ ) (B) Au NP agglomerates upon interaction with $E_{2}$ at $500 \mathrm{ng} / \mathrm{ml}$ while the inset shows a pink strip typical of $E_{2}$ at lower concentrations

The electrospun fibres were then cut into strips that were employed as colorimetric probes. The probe strips were exposed to a series of $17 \beta$-estradiol concentrations. Figure 5 presents a profile of the colorimetric response of the probe strips towards the various $\mathrm{E}_{2}$ concentrations. It was observed that with increasing $\mathrm{E}_{2}$ concentrations ( $50 \mathrm{ng} / \mathrm{m} \ell$ to $1000 \mu \mathrm{g} / \mathrm{m} \ell$ ) the colour of the probe changed gradually from white to shades of pink and eventually to shades of blue at higher $\mathrm{E}_{2}$ concentrations. With an increase in $\mathrm{E}_{2}$ concentration the surface plasmon resonance (SPR) band shifted to a longer wavelength (Fig. 5). The surface plasmon resonance band shift is usually accompanied by a visual colour change, from pink to blue.

It was also observed that upon interaction of the probe strip, even at $\mathrm{ng} / \mathrm{m} \ell 17 \beta$-estradiol concentrations, the clusters tended to agglomerate further and became more visible on the fibre surface. Figure 6A illustrates typical agglomeration of $\mathrm{Au}$ NPs upon interacting with $800 \mu \mathrm{g} / m \ell \mathrm{E}_{2}$, while the inset depicts a blue shade indicative of higher $\mathrm{E}_{2}$ concentrations. Figure $6 \mathrm{~B}$, on the other hand, is a typical SEM image showing that lower
$\mathrm{E}_{2}$ concentrations induced relatively less visible Au NP agglomerates (as shown by the white clumps). Figure 6B inset is an optical photograph for the probe showing a typical pink colour for lower $\mathrm{E}_{2}$ concentrations.

To evaluate the performance of PS-Au NP probes, the strips were applied to spiked dairy effluents that were analysed without any further treatment. Individual matrix samples were spiked with $200 \mathrm{ng} / \mathrm{ml}$ of $\mathrm{E}_{2}$ as well as with cholesterol, p,p'-DDE, deltamethrin, 4-tert-octylphenol and nonylphenol, as these compounds are known to induce oestrogenic activity. Although cholesterol does not have oestrogenic activity it has been found to be at higher concentrations relative to oestrogens, in effluent from wastewater treatment plants (Oishi, 2010). 17 $\beta$-estradiol and cholesterol were therefore selected as representatives of oestrogens and other steroids, respectively. It was observed that the probe changed from white to a brown shade on exposure to the unspiked matrix. Upon interacting with the different analytes the probe strip turned pink with $\mathrm{E}_{2}$, while with p,p'-DDE, deltamethrin, 4-tert-octylphenol, 


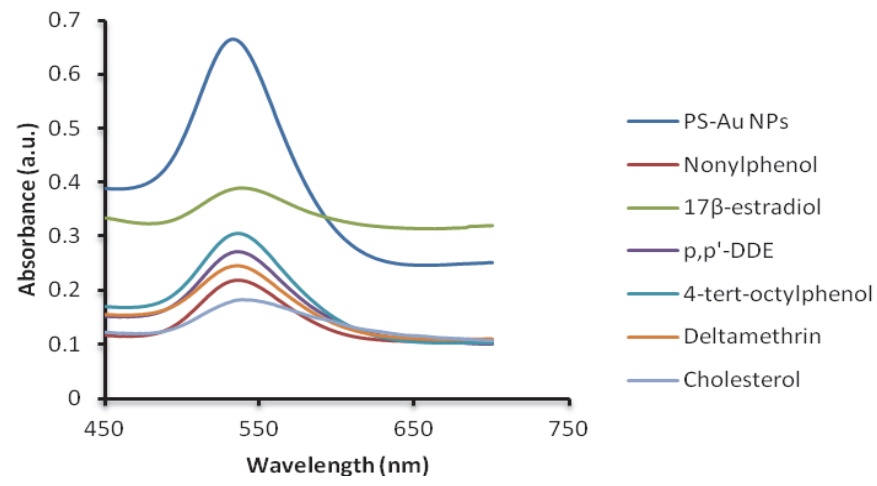

Figure 7

UV-vis absorbance spectra and photographs showing colorimetric responses of strips upon exposure to $200 \mathrm{ng} / \mathrm{ml} 17 \beta$-estradiol, $p, p^{\prime}-D D E$, deltamethrin, 4-tertoctylphenol, nonylphenol and cholesterol for 120 s at ambient temperature

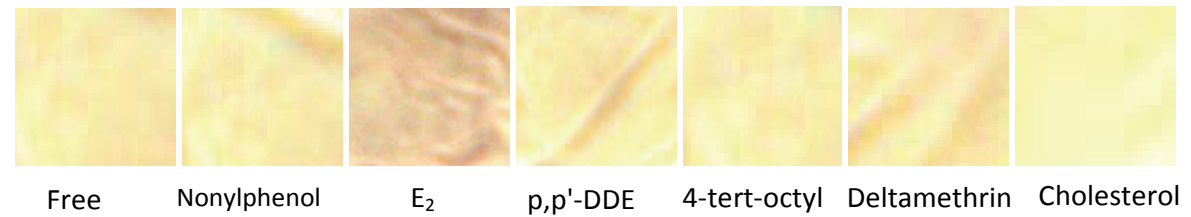

nonylphenol and cholesterol the probe showed a similar brownish colour as with the free matrix (Fig. 7). The probe strips were exposed to the analytes for $120 \mathrm{~s}$. Considering the observed colour changes, the developed probe showed good selectivity towards $\mathrm{E}_{2}$.

\section{CONCLUSION}

An electrospun simple colorimetric probe based on PS-Au NPs was developed. Taking advantage of several fascinating features of the PS-Au NP combination, the probe showed an excellent response towards $17 \beta$-estradiol and possessed high sensitivity, with a lowest naked-eye detection limit of $100 \mathrm{ng} / \mathrm{ml}$. This colorimetric system does not employ any sophisticated instrumentation, therefore it guarantees user-friendly, rapid and on-site detection of $17 \beta$-estradiol. Furthermore, relative to other nanoparticle-based colorimetric assays, this probe does not entail the use of complex nanoparticle modification (e.g. biological material). The probe warrants further investigation as it has shown potential to profile oestrogenic compounds in aqueous environments. As a way forward, a thiol-functionalised polystyrene may be used to enhance stability of the probe in harsh environmental conditions that include a wide range of temperature, concentration and $\mathrm{pH}$.

\section{ACKNOWLEDGEMENTS}

We acknowledge financial support from the Water Research Commission, South Africa, and also express our gratitude towards Nelson Mandela Metropolitan University (NMMU), Physics Department, for allowing us to use their high resolution SEM.

\section{REFERENCES}

BAI J, LI Y, LI M, WANG S, ZHANG C and YANG Q (2008) Electrospinning method for the preparation of silver chloride nanoparticles in PVP nanofiber. Appl. Surf. Sci. 254 4520-4523.

CHIGOME S and TORTO N (2011) A review of opportunities for electrospun nanofibers in analytical chemistry. Anal. Chim. Acta $70625-36$.

CHIU T-C, CHANG L-C, CHIANG C-K and CHANG H-T (2008) Determining estrogens using surface-assisted laser desorption/ ionization mass spectrometry with silver nanoparticles as the matrix. J. Am. Soc. Mass Spectrom. 19 1343-1346.

DING N, ZHAO H, PENG W, HE Y, ZHOU Y, YUAN L and ZHANG Y (2012) A simple colorimetric sensor based on anti-aggregation of gold nanoparticles for $\mathrm{Hg}^{2+}$ detection. Colloids Surf. A 395 161-167.

GADD JB, TREMBLAY LA and NORTHCOTT GL (2010) Steroid estrogens, conjugated estrogens and estrogenic activity in farm dairy shed effluents. Environ. Pollut. 158 730-736.

GAO J, HUANG X, LIU H, ZAN F and REN J (2012) Colloidal stability of gold nanoparticles modified with thiol compounds: bioconjugation and application in cancer cell imaging. Langmuir $\mathbf{2 8}$ 4464-4471.

GRUBBS RB (2007) Roles of polymer ligands in nanoparticle stabilization. Polymer Rev. 47 197-215.

HANSELMAN TA, GRAETZ DA and WILKIE AC (2003) Manureborne estrogens as potential environmental contaminants: A review. Environ. Sci. Technol. 37 5471-5478.

HANSELMAN TA, GRAETZ DA, WILKIE AC, SZABO NJ and DIAZ CS (2006) Determination of steroidal estrogens in flushed dairy manure wastewater by gas chromatography-mass spectrometry. J. Environ. Qual. 35 695-700.

JANS H and HUO Q (2012) Gold nanoparticle-enabled biological and chemical detection and analysis. Chem. Soc. Rev. 41 2849-2866.

JONES MR, OSBERG KD, MACFARLANE RJ, LANGILLE MR and MIRKIN CA (2011) Templated techniques for the synthesis and assembly of plasmonic nanostructures. Chem. Rev. 111 3736-3827.

KIM JK \& AHN H (2008) Fabrication and characterization of polystyrene/gold nanoparticle composite nanofibers. Macromol. Res. 16 $163-168$.

LU X, ZHAO Y and WANG C (2005) Fabrication of PbS nanoparticles in polymer-fiber matrices by electrospinning. Adv. Mater. 17 2485-2488.

MA Y, NIU H, ZHANG X and CAI Y (2011) One-step synthesis of silver/dopamine nanoparticles and visual detection of melamine in raw milk. Analyst 136 4192-4196.

MAYER ABR (1998) Formation of noble metal nanoparticles within a polymeric matrix: nanoparticle features and overall morphologies. Mater. Sci. Eng. C 6 155-166.

OISHI M (2010) Removal of dissolved estrogen in sewage effluents by $\beta$-cyclodextrin polymer. Sci. Total Environ. 409 112-115.

OLENIN Y and LISICHKIN GV (2011) Metal nanoparticles in condensed media: preparation and the bulk and surface structural dynamics. Russ. Chem. Rev. 80 605-630.

ROMEO HE, VÍLCHEZ A, ESQUENA J, HOPPE CE and WILLIAMS RJJ (2012) Polymerization-induced phase separation as a one-step strategy to self-assemble alkanethiol-stabilized gold nanoparticles inside polystyrene domains dispersed in an epoxy matrix. Eur. Polym. J. 48 1101-1109. 
SAQUING CD, MANASCO JL and KHAN SA (2009) Electrospun nanoparticle-nanofiber composites via a one-step synthesis. Small 5 944-951.

SEO J-S, SON D-M, LEE H, KIM J and KIM Y (2009) The characterization of borohydride-stabilized nanosilvers in laponite sol using 1H NMR: Its ligand exchange reactions with MUA and TOP. Bull. Korean Chem. Soc. 302651.

UEHARA N (2010) Polymer-functionalized gold nanoparticles as versatile sensing materials. Anal. Sci. 26 1219-1228.

WANG C, XU C, CHEN F and TANG X (2011) Simultaneous determination of three naturally occurring estrogens in environmental waters by high-performance liquid chromatography. J. Sep. Sci. 34 2371-2375.
ZHANG M, LIU Y-Q and YE B-C (2011) Colorimetric assay for sulfate using positively-charged gold nanoparticles and its application for real-time monitoring of redox process. Analyst 136 4558-4562.

ZHANG Y, LI B and XU C (2010) Visual detection of ascorbic acid via alkyne-azide click reaction using gold nanoparticles as a colorimetric probe. Analyst 135 1579-1584.

ZHAO W, BROOK MA and LI Y (2008) Design of gold nanoparticlebased colorimetric biosensing assays. ChemBioChem 9 2363-2371.

ZHENG W, YATES SR and BRADFORD SA (2008) Analysis of steroid hormones in a typical dairy waste disposal system. Environ. Sci. Technol. 42 530-535. 